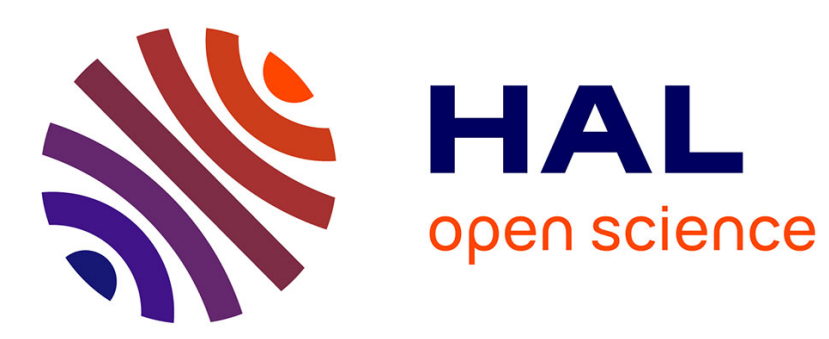

\title{
Force Variations Related to Flow Pattern Changes Around a High-Camber Thin Wing
}

Patrick Bot

\section{To cite this version:}

Patrick Bot. Force Variations Related to Flow Pattern Changes Around a High-Camber Thin Wing. AIAA Journal, 2019, pp.1-7. 10.2514/1.j058443 hal-02470868

\section{HAL Id: hal-02470868 \\ https://hal.science/hal-02470868}

Submitted on 7 Feb 2020

HAL is a multi-disciplinary open access archive for the deposit and dissemination of scientific research documents, whether they are published or not. The documents may come from teaching and research institutions in France or abroad, or from public or private research centers.
L'archive ouverte pluridisciplinaire HAL, est destinée au dépôt et à la diffusion de documents scientifiques de niveau recherche, publiés ou non, émanant des établissements d'enseignement et de recherche français ou étrangers, des laboratoires publics ou privés. 


\title{
Force Variations Related to Flow Pattern Changes Around a High-Camber Thin Wing
}

\author{
P. Bot* \\ Naval Academy Research Institute, 29200 Brest, France
}

https://doi.org/10.2514/1.J058443

\begin{abstract}
The aim of this paper is to report and analyze experimental results on forces generated by a high-camber thin section with a sharp leading edge, namely, a curved plate with $22.3 \%$ relative camber, and measurements of the flowfield, in a nominally 2D flow. A wide range of angles of attack $\left[-10^{\circ} ; 3^{\circ}\right]$ is investigated for 3 values of the Reynolds number across the transitional range. Unusual polar curves are observed with sharp variations of lift with the angle of attack. At low incidence, the lift coefficient increases faster than $2 \pi /$ radian. Moreover, very different behaviors occur for Reynolds numbers lower or higher than $\sim 10^{5}$. For small or negative angles of attack, the lift coefficient is negative (downward lift) at small Reynolds numbers, but positive (upward lift) at higher Reynolds numbers. The velocity field is investigated with particle image velocimetry. Variations of lift are related to changes in the topology of the flowfield around the section. These results help to better understand the flow around a high-camber thin section and provide a detailed benchmark database in a simple geometry for model validations. As strong Reynolds number effects are evidenced, this database would be particularly useful to assess the performance of transition models.
\end{abstract}

\section{Nomenclature}

$C_{D}=$ drag coefficient

$C_{L}=$ lift coefficient

$c=$ chord length, $\mathrm{m}$

$D=$ drag force, $\mathrm{N}$

$L=$ lift force, $\mathrm{N}$

$R e=$ Reynolds number, $U_{0} c / \nu$

$s \quad=$ profile span, $\mathrm{m}$

$U_{0}=$ upstream velocity, $\mathrm{m} / \mathrm{s}$

$\alpha=$ angle of attack, ${ }^{\circ}$

$\alpha_{r} \quad=\quad$ angle of attack; $\alpha \pi / 180, \mathrm{rad}$

$\rho \quad=\quad$ fluid density, $\mathrm{kg} / \mathrm{m}^{3}$

$\nu=$ fluid kinematic viscosity, $\mathrm{m}^{2} / \mathrm{s}$

\section{Introduction}

I $\mathrm{N}$ AERONAUTICS, most studies are focused on relatively thin airfoil sections with a moderate camber, which are the most appropriate when the objective is to maximize the lift-to-drag ratio. In certain circumstances, however, such as a landing aircraft or a yacht downwind sail, for example [1, 2], the objective is rather to maximize lift, and a high drag is not a penalty or may even be an advantage. In such a case, a high-camber section may be used with a high angle of attack. The flow around these not-so-slender bodies is complex because of large flow separation, and their simulation with computational fluid dynamics is rather challenging [3] . Hence, detailed experimental data are desirable to better understand the physics of such flows and to compare with simulation results. Real 3D flows are even more complex.

Moreover, traditional wing sections have a rounded leading edge in order to avoid flow separation at the leading edge in a reasonable range of angle of attack. Certain applications, such as thin membrane wings, for instance, have a sharp leading edge, which results in flow separation right at the leading edge as soon as the incoming flow is not locally aligned with the section's entry (often referred to as the ideal angle of attack). Furthermore, many studies devoted to lowReynolds-number (low-Re) flows have shown that thin sections with

\footnotetext{
*Associate Professor, Mechanical Engineering, CC600, Cedex 9; patrick .bot@ecole-navale.fr.
}

sharp leading edges outperform classical high- $R e$ wings (see, e.g., $[4,5])$. For applications to micro air vehicles, better rotor performances were obtained with sharp and thin plates bent to a circular arc with low or moderate camber (see, e.g., [6,7]).

In the present study, a 2D extruded section of a circular arc with a sharp leading edge - a curved plate with chamfered ends - is tested in order to investigate the lift and drag forces and the flowfield in a simple geometry and better understand this kind of flow, where strong separation occurs. In particular, strong $R e$ effects are highlighted and these experimental results may prove helpful to assess the performance of transition models (see, e.g., [ㅇ-10]). Concerning practical applications, a curved plate is an easy-to-manufacture high-lift section, which might be used for wind or tidal energy harvesting [11].

In the literature, many previous works have been done on cambered plates with low or moderate camber (less than $10 \%$ relative camber) at low $R e$, typically in the context of insect wing aerodynamics and micro air vehicles (see, e.g., [느-7 $, 12,13])$. However, studies of highly cambered plates (more than $20 \%$ relative camber) are much more limited in the literature, and particularly in the present range of $\operatorname{Re}\left(10^{4}-10^{5}\right)$ and there is no general understanding of the aero/ hydrodynamics in such a geometry. In 1971, Milgram [14] reported experimental section data for thin, cambered plates with cambers up to $20 \%$. He showed that the slope of the lift curve is higher than $2 \pi /$ radian for $\alpha$ lower than the ideal angle of attack, and smaller than $2 \pi /$ radian for $\alpha$ higher than ideal. Milgram anticipated that this should be due to flow separation on the pressure side (resp. on the suction side) for $\alpha$ lower (resp. higher) than ideal, but no investigation of the flowfield was provided. Classical wing sections with rounded nose and low camber are well known to generate a lift coefficient increasing almost as $2 \pi \alpha_{r}$ until a sharp drop occurs when stall is reached at a high angle of attack, around $10-20^{\circ}$ depending on the section details (for recent work, see, e.g., $[15,16])$. For thin, highly cambered sections with a sharp leading edge, the lift coefficient remains smaller than $2 \pi \alpha_{r}$, but no sudden stall is observed. Once again, it is suspected that this is because substantial flow separation exists at all angles. As a consequence, the minimum drag is one order of magnitude higher than that on classical wing sections.

Later, Collie [3] and Collie et al. [17] investigated a curved plate with a camber of $24.7 \%$ as a simplified geometry of a yacht downwind sail. The measured lift coefficient curve shows a local maximum at $\alpha=10^{\circ}$, which is considered to be the ideal angle of attack. The observed lift drop between $\alpha=10$ and $15^{\circ}$ is supposed to be associated with the appearance of a leading edge separation detected from computational fluid dynamics. However, the simulation results are very sensitive to the turbulence model and do not match the experimental data very well. Above $15^{\circ}$, the lift coefficient increases 
again. Interestingly, a recent study [18] considered the flow at $R e=1.5 \times 10^{4}$ around a high-camber plate with a geometry very similar to the present study (chamfered ends, relative camber of $29 \%$ ), as a simplified cross section of a flying snake (Chrysopelea paradisi) to gain insight into its unexpected gliding performance in the absence of wings (see also [19] in a slightly different geometry closer to the actual snake cross section and for $R e$ between $3 \times 10^{3}$ and $1.5 \times 10^{4}$ ). Their results show a high lift and sustained lift-to-drag ratio on a large range of angles of attack, and particularly a spike in the lift coefficient at $\alpha=30^{\circ}$. Moreover, their results proved rather robust to small variations in the shape of the section.

Recently, Bot et al. [20] showed that the curved plate studied in the present paper, when placed at $\alpha=0^{\circ}$ exhibits a sharp jump of lift when the $R e$ exceeds $2 \times 10^{5}$ : the downward lift flips to an upward lift. Simulations in [8-10] reproduced the phenomenon. This effect is related to the transition in the suction side boundary layer, which delays the separation from the convex wall and was named a "lift crisis," by analogy to the drag crisis well known on bluff bodies.

The present work reports hydrodynamic force measurements and flow visualizations with particle image velocimetry (PIV) around a circular arc plate in nominally 2D flow to relate the forces to the associated flow patterns. Indeed, as highlighted, for example, in [21], the joint analysis of forces and flowfield proves very helpful to get a better insight into the physics of the flow and may be useful in the perspective of flow control [22]. The tested section, the experimental setup, and data processing are presented in Sec. II. Section III presents the results on forces and flow patterns, which are discussed in Sec. IV. Conclusions and perspectives are given in Sec. $\underline{\mathrm{V}}$.

\section{Experimental Setup and Data Processing}

Experiments were carried out in the French Naval Academy hydrodynamic tunnel (Fig. 1) to measure the forces and the velocity fields on a $2 \mathrm{D}$ high-camber plate. The plate is a 3-mm-thick, 50-mmradius stainless steel circular arc section with a chord length $c=$ $74.45 \mathrm{~mm}$ and a camber $t=16.6 \mathrm{~mm}$, resulting in a relative camber $t / c=22.3 \%$ located at midchord (Fig. 2 and Table 1 ). This geometry is inspired from a horizontal section of a typical yacht downwind

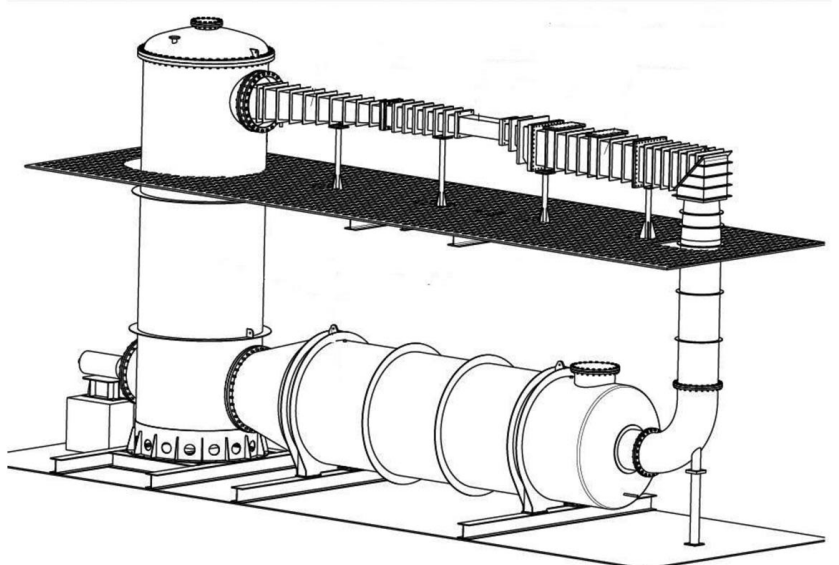

Fig. 1 IRENav hydrodynamic tunnel.

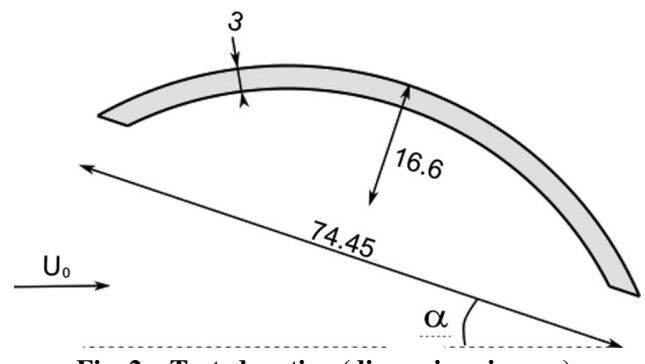

Fig. 2 Tested section (dimensions in $\mathbf{m m}$ ).
Table 1 Profile characteristics

\begin{tabular}{lc}
\hline \hline Chord length $c, \mathrm{~mm}$ & 74.45 \\
Span $s, \mathrm{~mm}$ & 191 \\
Maximum camber, mm & 16.62 \\
Relative camber $(\%$ of $c$ ) & 22.32 \\
Leading and trailing edge angle, $^{\circ}$ & 48.12 \\
Maximum camber position: draft $(\%$ of $c$ ) & 50 \\
Aspect ratio $(s / c)$ & 2.57 \\
\hline \hline
\end{tabular}

sail [1]. The test section is $192 \times 192 \mathrm{~mm}^{2}$ and $1 \mathrm{~m}$ long, located downstream of honeycombs and a $1 / 9$ contraction convergent. The measured turbulence intensity is $1.8 \%$. The section span fills almost all the channel width, except for a small gap on each end to avoid contacts with the walls that would alter force measurements. The profile is mounted at midheight of the test section (Fig. 3), cantilevered on a strain gauge hydrodynamic balance to measure the forces. The balance is fixed on a rotating frame mounted on bearings and driven in rotation by a stepper motor with $6 \times 10^{5}$ impulsions per revolution. Angle of attack is controlled by a home-made LabVIEW interface with accuracy better than $0.1^{\circ}$. The balance strain gauges are controlled by an IOtech Strainbook 616, and forces are determined thanks to a previous calibration with accuracy better than $1.7 \mathrm{~N}$ for the lift and $0.5 \mathrm{~N}$ for the drag. The related absolute uncertainties for $C_{L}$ (resp. $C_{D}$ ) range from 0.009 (resp. 0.003) for $R e=3.69 \times 10^{5}$ up to 0.2 (resp. 0.07) for $R e=6.82 \times 10^{4}$.

Forces are recorded at $1 \mathrm{kHz}$, and results presented here are time averaged during $30 \mathrm{~s}$. More details on the force balance and mounting can be found in [23]. The lift and drag coefficients are defined as

$$
C_{L}=\frac{L}{1 / 2 \cdot \rho \cdot c \cdot s \cdot U_{0}^{2}} \quad C_{D}=\frac{D}{1 / 2 \cdot \rho \cdot c \cdot s \cdot U_{0}^{2}}
$$

Velocity field measurements are performed around the obstacle using 2D PIV. The flow is illuminated from above with a pulsed laser sheet normal to the spanwise direction and located at midspan. Two mirrors are placed below the tunnel test section to illuminate the other side of the plate, which allows for the whole velocity field around the obstacle to be measured at once. The light sheet thickness is around $2 \mathrm{~mm}$ in the recorded flowfield. The water flow is seeded with micrometer-sized polyamide particles and visualized by a $4 \mathrm{k}$-pixel charge-coupled device camera at a sampling frequency of $10 \mathrm{~Hz}$ in double frame. For each value of $\alpha$ and $R e, 300$ image pairs are recorded and processed with Dantec's DynamicStudio in order to compute 300 snapshots of the instantaneous flowfield, from which the time-averaged velocity field $U(x, y)$ is determined. Raw images are preprocessed the following way: the time-averaged image is subtracted to eliminate the light flare on the section walls, and the resulting images are normalized by the time-averaged image to mitigate illumination inhomogeneity. Preprocessed image pairs are then processed with the AdaptivePIV algorithm [24]. The final interrogation window is $32 \times 32$ pixels $^{2}$ with a $50 \%$ overlap, yielding a spatial resolution of $1.25 \mathrm{~mm}(0.017 c)$, and typically 3 correlation passes are processed. Considering the whole measurement process, the measurement uncertainty on velocity is estimated to be around $1 \%$ of the freestream velocity. The minimal distance to the section walls for a correct velocity measurement varies from almost $1 \mathrm{~mm}$ $(0.013 c)$ in the image center up to $2 \mathrm{~mm}(0.026 c)$ away from the image center due to perspective effects, as the section tip hides the wall in the midspan PIV plane.

Measurements were achieved for the $R e$ values $6.82 \times 10^{4}$, $2.38 \times 10^{5}$, and $3.69 \times 10^{5}$ and sweeping angle of attack from $-10^{\circ}$ up to $32^{\circ}$. The used procedure for both force and PIV measurements is a step-by-step increase of the angle of attack with continuously running water tunnel, with at least $1 \mathrm{~min}$ delay before each measurement.

The time-averaged vector map is used to determine the flow pattern, which is visualized by drawing the streamlines. Then, the 


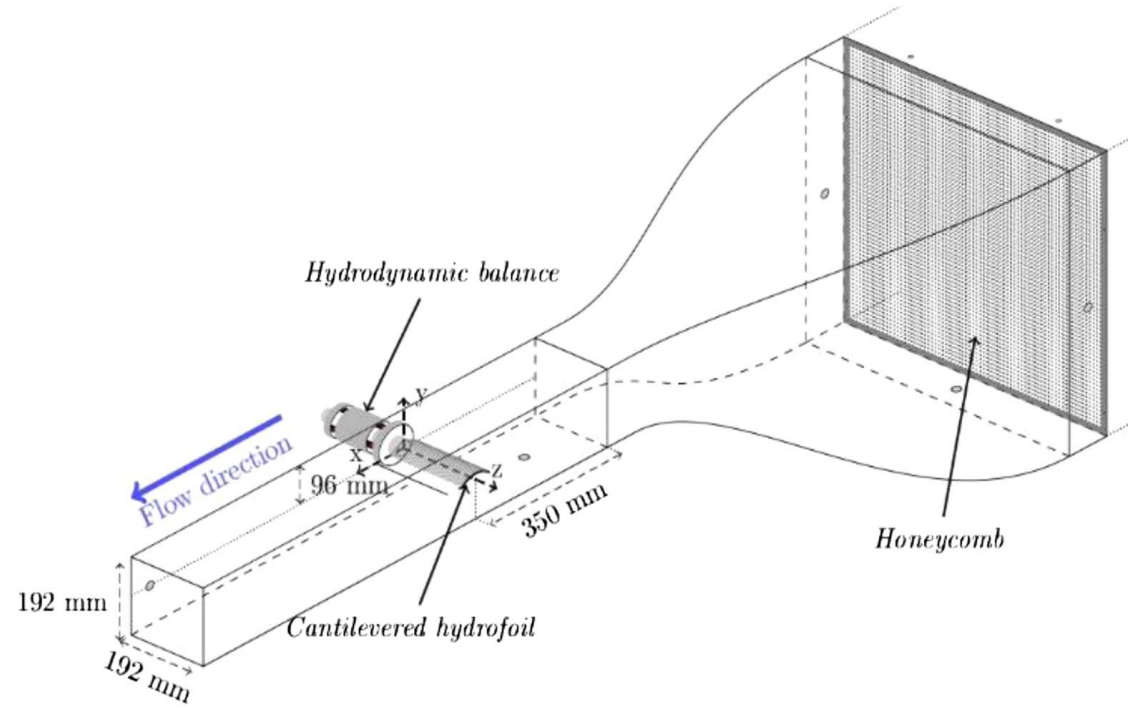

Fig. 3 Test section of the tunnel and position of the tested profile.

mean flow topology is characterized for all measured angles and $R e$ values, and changes in the flow pattern are related to variations of the lift and drag generated by the section. The streamlines are the easiest way to figure out the global flow pattern. Refined details such as flow separation location are determined more accurately with a thorough investigation of the vector maps with a large zoom and plotting all vectors (unlike in Figs. $\underline{4}$ and $\underline{5}$ ) scrolling the regions of interest.

Note that the actual flow is unsteady, particularly when large-scale vortices are shed in the wake when massive flow separation occurs. The low-speed PIV used here allows for a high spatial resolution, but does not allow for resolving the typical time scales of the flow fluctuations (in the range $20-100 \mathrm{~Hz}$ ). Hence, only the time-averaged velocity fields are analyzed here. Examples of instantaneous velocity fields and fluctuations of velocity components are shown Figs. $\underline{4}$ and 5. Further work is under way to investigate the dynamic behavior thanks to a high-frequency PIV package.

\section{Results}

\section{A. Lift and Drag Coefficients}

Figure 6 shows variations of the lift coefficient with angle of attack for the three $R e$ values tested. It is noticeable that the behavior is rather similar for the two highest $R e$ and very different from the one observed at the lowest $R e$. For $R e=6.82 \times 10^{4}, C_{L}$ is negative-downward lift-for $\alpha$ below $3^{\circ}$, and increases with a slope higher than $2 \pi /$ radian up to $6^{\circ}$ and then a slope lower than $2 \pi$ /radian up to $14^{\circ}$. A sharp jump occurs at $\alpha=15^{\circ}$ and the lift coefficient levels off around 2.15 for the highest angles. For $R e=2.38 \times 10^{5}$ and $R e=3.69 \times 10^{5}$, the lift coefficient $C_{L}$ is always positive - upward lift-whatever the angle of attack in the tested range and remains much higher than for $R e=6.82 \times 10^{4}$, up to $\alpha=15^{\circ}$. Another striking feature of the two highest $R e$ curves is that after almost linearly increasing from $\alpha=-2^{\circ}, C_{L}$ smoothly drops after $\alpha=6$ or $8^{\circ}$, and bounces back up for $\alpha \geq 10^{\circ}$ or $11^{\circ}$.
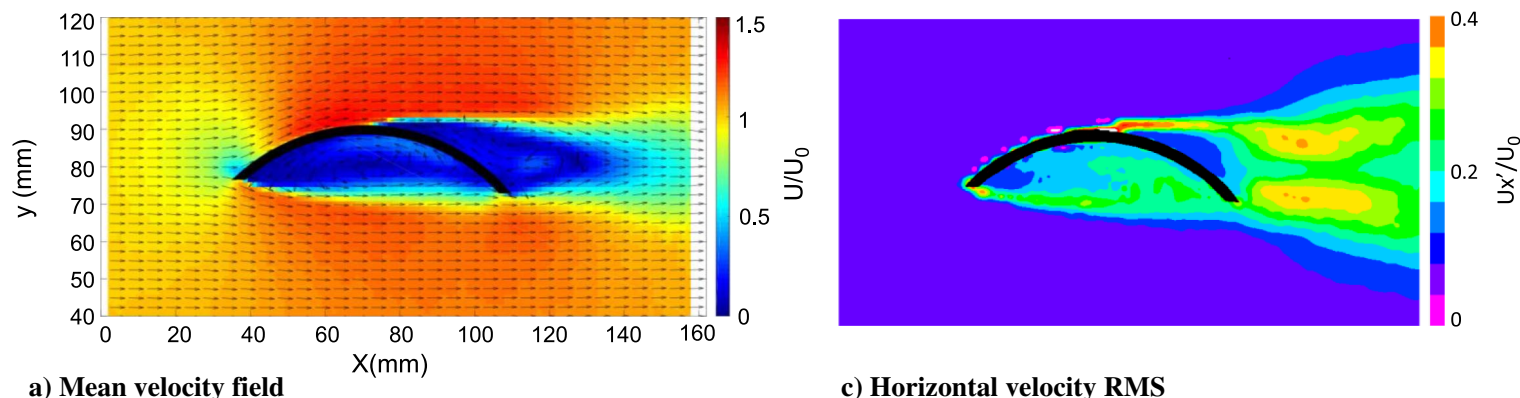

c) Horizontal velocity RMS
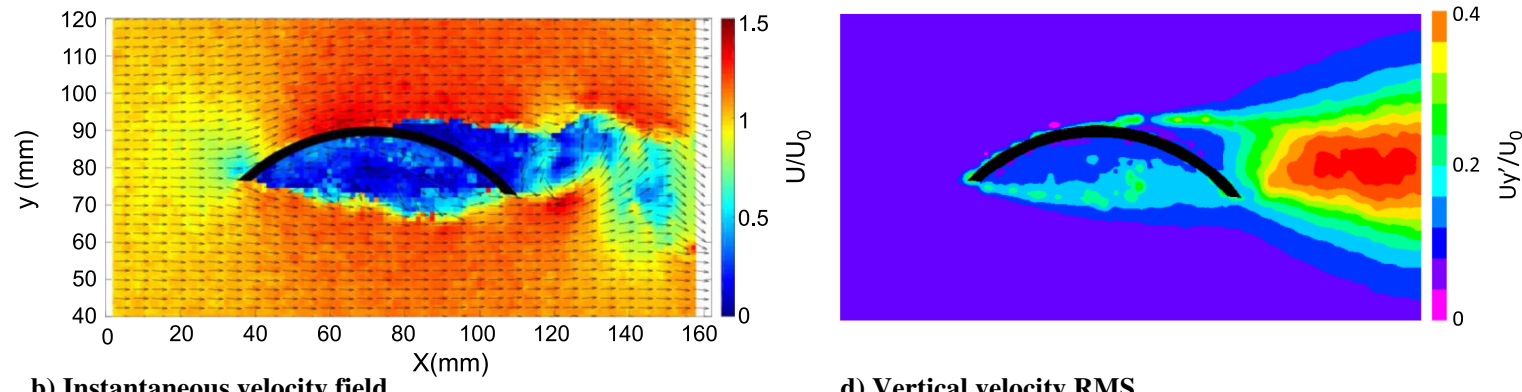

b) Instantaneous velocity field

d) Vertical velocity RMS

Fig. 4 Time-averaged velocity field (a), snapshot of instantaneous velocity field (b), standard deviation of the horizontal velocity component (c), standard deviation of the vertical velocity component (d), for $\alpha=4^{\circ}$ and $R e=6.82 \times 10^{4}$. Flow is from left to right. On the velocity fields (a and b), only one vector every eight is shown for clarity, and all vectors are of unit length; the nondimensional velocity magnitude $U / U_{0}$ is shown by the color scale. 


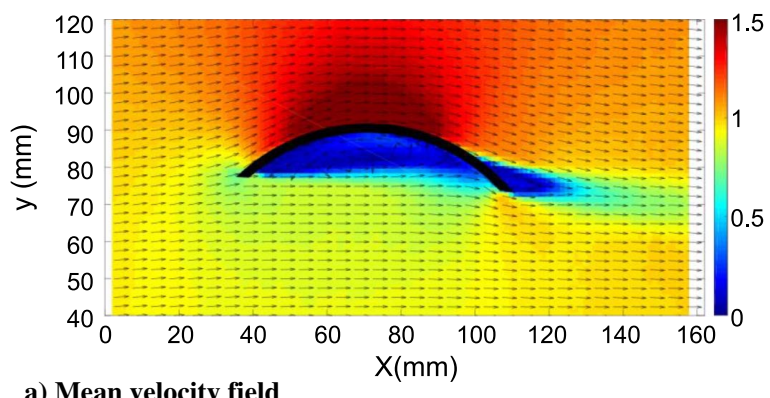

a) Mean velocity field

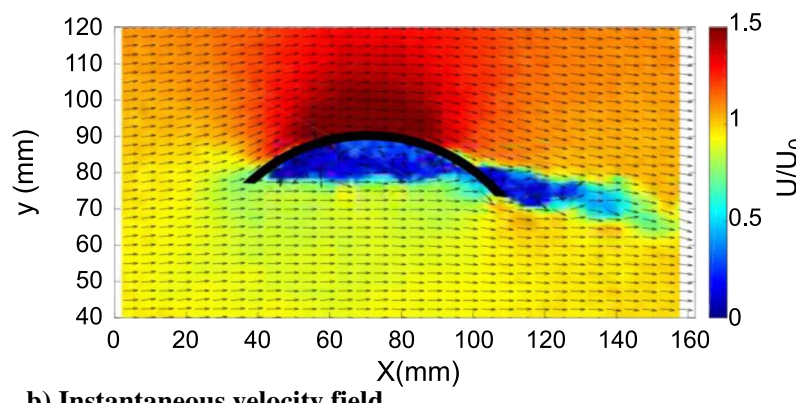

b) Instantaneous velocity field

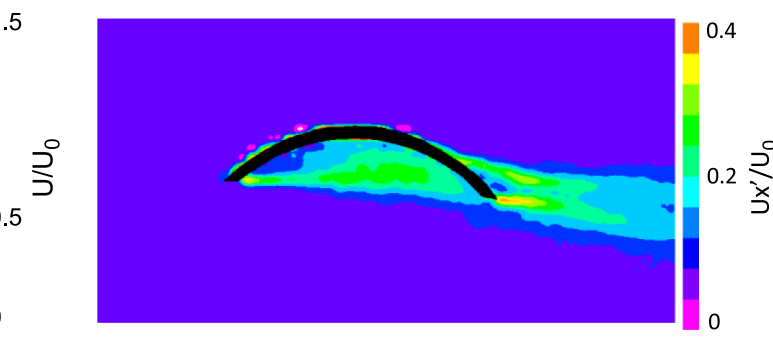

c) Horizontal velocity RMS

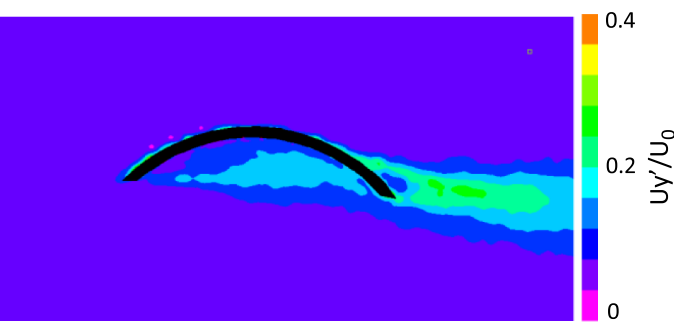

d) Vertical velocity RMS

$\operatorname{Re}=3.6910^{5} ; \alpha=4^{\circ}$

Fig. 5 Time-averaged velocity field (a), snapshot of instantaneous velocity field (b), standard deviation of the horizontal velocity component (c), standard deviation of the vertical velocity component (d), for $\alpha=4^{\circ}$ and $R e=3.69 \times 10^{5}$. Flow is from left to right. On the velocity fields (a and b), only one vector every eight is shown for clarity, and all vectors are of unit length; the nondimensional velocity magnitude $U / U_{0}$ is shown by the color scale.

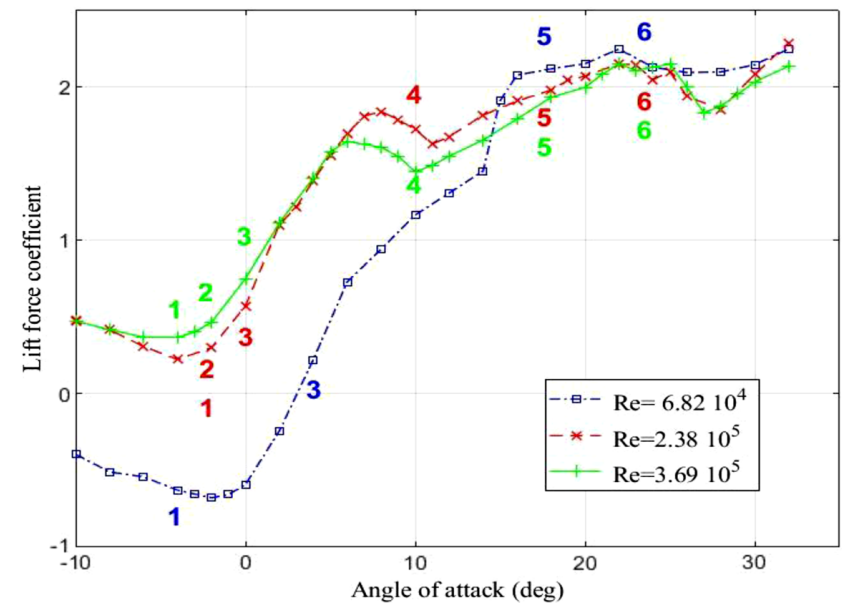

Fig. 6 Lift coefficient variations with angle of attack for different Reynolds numbers. Numbers labeled refer to changes in the flow pattern listed in Table 2 and shown in Fig. $\underline{8}$.

A second minimum is found around $\alpha \approx 27-28^{\circ}$. It should be noted that, for angles higher than $25^{\circ}$, the lift fluctuates quite a lot in time and the section is subject to significant vibrations, particularly for the highest values of $R e$.

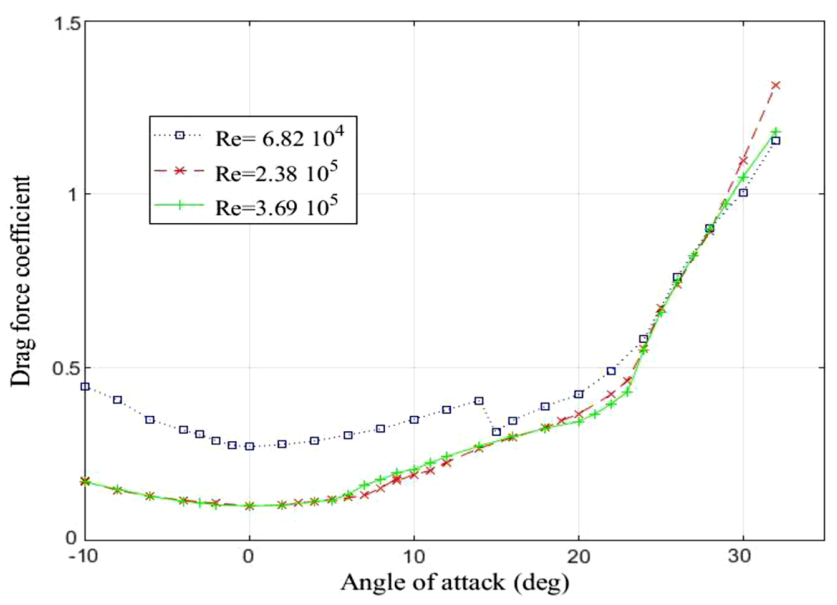

Fig. 7 Drag coefficient variations with angle of attack for different Reynolds numbers. Note that the uncertainty on the drag coefficient for $R e=6.82 \times 10^{4}$ is very high (of order $100 \%$ ) because the drag force is very low.

Variations of the drag coefficient with angle of attack are shown in Fig. 7 for the three $R e$ values tested. Once again, results are very similar for the two highest values of $R e$ and very different from the

Table 2 Topological changes in the mean flow pattern shown in Fig. $\underline{8}$ and labeled in Fig. $\underline{6}$

\begin{tabular}{|c|c|c|c|c|}
\hline \multirow[b]{2}{*}{ S. No. } & \multirow[b]{2}{*}{ Topology change } & \multicolumn{3}{|c|}{$\operatorname{Re}$} \\
\hline & & $6.82 \times 10^{4}$ & $2.38 \times 10^{5}$ & $3.69 \times 10^{5}$ \\
\hline 1 & Onset of secondary vortex in front of recirculation inside lower wall & $\alpha=-4^{\circ}$ & $\alpha=-2^{\circ}$ & $\alpha=-4^{\circ}$ \\
\hline 2 & Onset of higher trailing edge vortex (clockwise) & All $\alpha$ & $\alpha=-2^{\circ \mathrm{a}}$ & $\alpha=-2^{\circ \mathrm{b}}$ \\
\hline 3 & $\begin{array}{l}\text { Onset of rear stagnation point on lower wall and vanishing } \\
\text { of secondary vortex in front of recirculation inside lower wall }\end{array}$ & $\alpha=4^{\circ}$ & $\alpha=0^{\circ}$ & $\alpha=0^{\circ}$ \\
\hline 4 & Onset of lower trailing edge vortex (anticlockwise) & All $\alpha^{\mathrm{c}}$ & $\alpha=10^{\circ}$ & $\alpha=10^{\circ}$ \\
\hline 5 & Vanishing of recirculation inside lower wall & $\alpha=18^{\circ}$ & $\alpha=18^{\circ}$ & $\alpha=18^{\circ}$ \\
\hline 6 & Deep stall (separation on upper wall reaches the leading edge) & $\alpha=24^{\circ}$ & $\alpha=24^{\circ}$ & $\alpha=24^{\circ}$ \\
\hline
\end{tabular}


lower $R e$. For $R e=6.82 \times 10^{4}$, the drag coefficient is much higher than at higher $R e$ until it suddenly drops to similar vales at $\alpha=15^{\circ}$. The drag coefficient is minimum for $\alpha \approx 0^{\circ}$ and the slope increases sharply when $\alpha$ exceeds $24^{\circ}$. For the lowest $R e$, the drag at $\alpha=0^{\circ}$ corresponds to a drag coefficient based on the frontal area close to 1 , corresponding to a fully separated flow behind the equivalent bluff body.

\section{B. Mean Flow Patterns}

The results of the PIV measurements are presented in Fig. $\underline{8}$ as streamline maps in the spanwise-normal plane of the time-averaged velocity field around the section at midspan, to highlight the changes in the flow pattern with angle of attack, for the different $R e$ 's tested. As a general observation, flow patterns for $R e=2.38 \times 10^{5}$ and $R e=3.69 \times 10^{5}$ are very similar, but strongly differ from the pat-
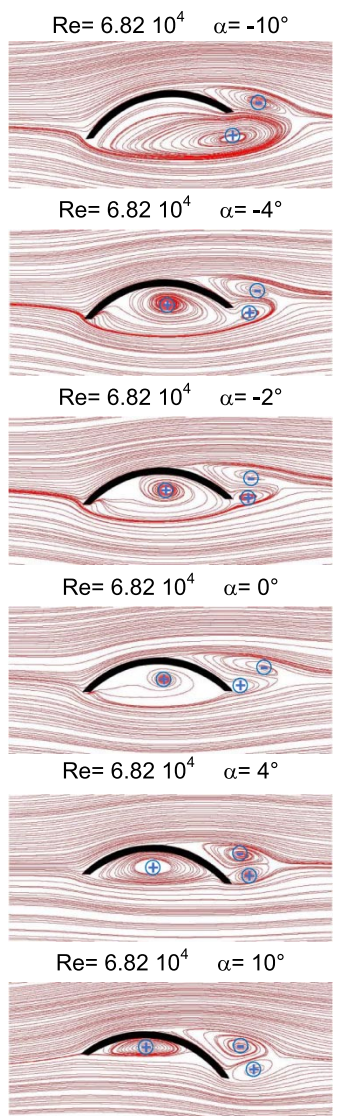

$\operatorname{Re}=6.8210^{4} \quad \alpha=14^{\circ}$

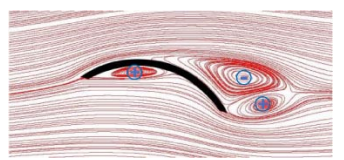

$\operatorname{Re}=6.8210^{4} \quad \alpha=18^{\circ}$

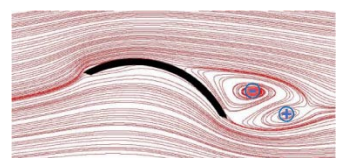

$\operatorname{Re}=6.8210^{4} \quad \alpha=22^{\circ}$

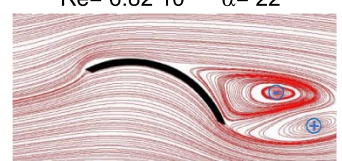

$\operatorname{Re}=6.8210^{4} \quad \alpha=26^{\circ}$

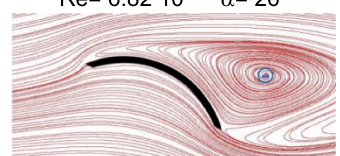

$\operatorname{Re}=6.8210^{4} \quad \alpha=32^{\circ}$

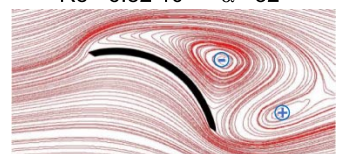

$\operatorname{Re}=2.3810^{5} \quad \alpha=10^{\circ}$

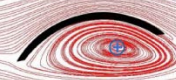

$\operatorname{Re}=2.3810^{5} \quad \alpha=-4^{\circ}$

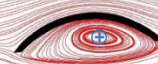

$\operatorname{Re}=2.3810^{5} \quad \alpha=-2^{\circ}$

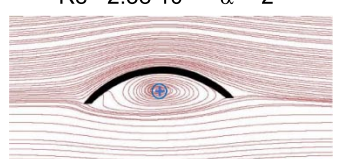

$\operatorname{Re}=2.3810^{5} \quad \alpha=0^{\circ}$

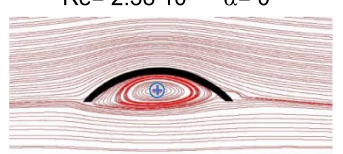

$\operatorname{Re}=2.3810^{5} \quad \alpha=4^{\circ}$

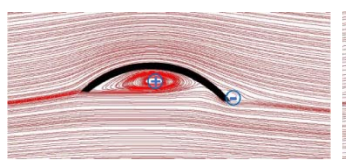

$\operatorname{Re}=2.3810^{5} \quad \alpha=10^{\circ}$

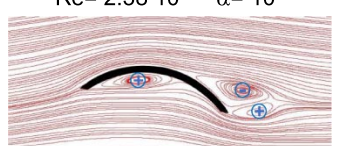

$R e=2.3810^{5} \quad \alpha=14^{\circ}$

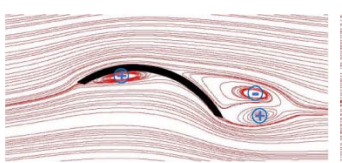

$\operatorname{Re}=2.3810^{5} \quad \alpha=18^{\circ}$

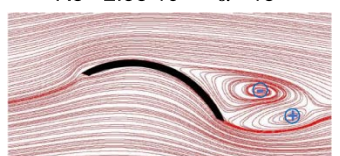

$\operatorname{Re}=2.3810^{5} \quad \alpha=22^{\circ}$

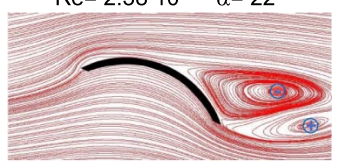

$\operatorname{Re}=2.3810^{5} \quad \alpha=26^{\circ}$

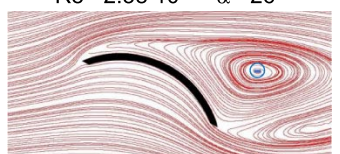

$\operatorname{Re}=2.3810^{5} \quad \alpha=32^{\circ}$

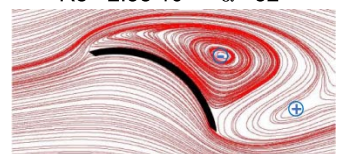

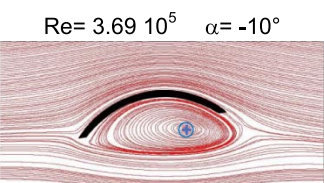

$\operatorname{Re}=3.6910^{5} \quad \alpha=-4^{\circ}$

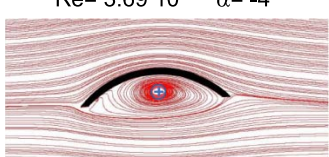

$\operatorname{Re}=3.6910^{5} \quad \alpha=2^{\circ}$

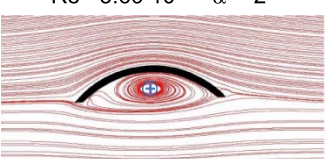

$\operatorname{Re}=3.6910^{5} \quad \alpha=0^{\circ}$

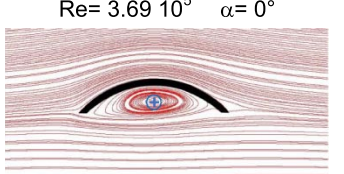

$\operatorname{Re}=3.6910^{5} \quad \alpha=4^{\circ}$

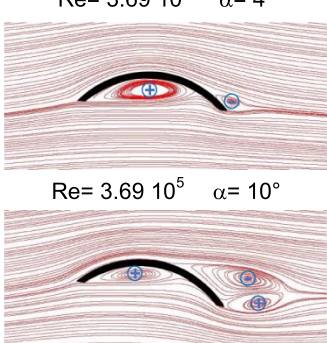

$R e=3.6910^{5} \quad \alpha=14^{\circ}$

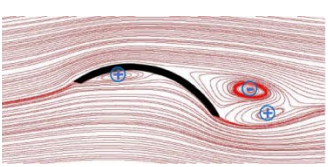

$\operatorname{Re}=3.6910^{5} \quad \alpha=18^{\circ}$

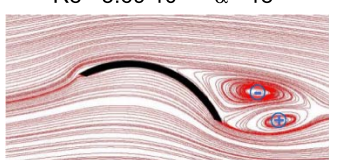

$\operatorname{Re}=3.6910^{5} \quad \alpha=22^{\circ}$

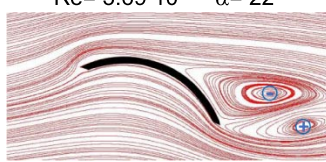

$\operatorname{Re}=3.6910^{5} \quad \alpha=26^{\circ}$

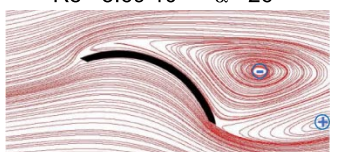

$\operatorname{Re}=3.6910^{5} \quad \alpha=32^{\circ}$

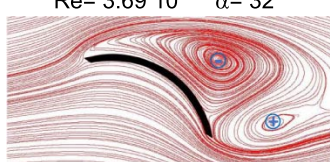

Fig. 8 Flow pattern in the tested range of angle of attack: time-averaged streamlines for $R e=6.82 \times 10^{4}$ (left), $R e=2.38 \times 10^{5}$ (middle), and $R e=3.69 \times 10^{5}$ (right). Flow is from left to right. Blue "+" symbols denote anticlockwise vortices, and blue "-"symbols denote clockwise vortices. 
terns observed at $R e=6.82 \times 10^{4}$ for angles lesser than around $10^{\circ}$. At this low $R e$, a large separation area exists on the upper surface whatever the angle of attack, and the section generates a wide wake with two contrarotating recirculation areas in average. For higher $R e$ on the contrary, the wake is much thinner at moderate angles with very little, if any, recirculation behind the body. More precisely, the separation area on the rear upper wall keeps shorter than around $0.2 c$ for $\alpha$ up to $8^{\circ}$, and a lower anticlockwise vortex shows up in the wake from the trailing edge when $\alpha$ reaches $10^{\circ}$. Then, for $\alpha \geq 10^{\circ}$, the time-averaged flow patterns are rather similar in the big picture for the different $R e$ values, with a pair of contrarotating vortices forming the recirculation area behind the section; despite that the wake remains larger at low $R e$. Complete stall occurs around $24^{\circ}$, where $C_{L}$ begins to drop and the separation location on the upper wall reaches the leading edge, giving rise to a massively separated flow dominated by the large clockwise vortex from the upper wall with a marked shear line issued from the trailing edge.

Concerning the concave wall, for low angles of attack, a large recirculation area takes place below the section. This recirculation extends much farther than the section chord line and the trailing edge at $\alpha=-10^{\circ}$, particularly for $R e=6.82 \times 10^{4}$, for which it forms the lower trailing edge (or wake) anticlockwise vortex, whereas the contrarotating upper wake vortex is formed by the rear separation on the upper wall. From $\alpha=0^{\circ}$ (high $R e$ ) or $4^{\circ}$ (low $R e$ ), a stagnation point appears on the lower wall near the trailing edge and the recirculation does not fill the whole cavity. This recirculation area below the concave wall progressively shrinks as the rear stagnation point moves toward the leading edge, until it totally disappears around $\alpha=18^{\circ}$.

For angles in the range from $-4^{\circ}$ up to $4^{\circ}\left(R e=6.82 \times 10^{4}\right)$ or up to $0^{\circ}\left(R e=2.38 \times 10^{5}\right.$ and $\left.R e=3.69 \times 10^{5}\right)$, a small clockwise secondary vortex is visible (on zoomed vector fields, not shown here) near the leading edge on the lower wall with a stagnation point right aft from the leading edge corner.

Finally, Figs. 4 and 5 show examples of the velocity field, both time averaged and a snapshot of instantaneous velocity field, along with the fluctuations of velocity components, in two representative cases, $\alpha=4^{\circ}, R e=6.82 \times 10^{4}$, and $R e=3.69 \times 10^{5}$. These examples clearly show the large difference in flow separation, wake width, and shed vortex size associated to these two values of $R e$ : massively separated flow and low lift at $R e=6.82 \times 10^{4}$, and mostly attached flow and high lift at $R e=3.69 \times 10^{5}$. More comprehensive sets of data are available upon request.

\section{Discussion}

The cambered plate investigated here is a geometrically simple realization of a high-lift section able to generate a lift coefficient as high as 2.2, at the expense of a rather high drag (maximum lift-to-drag ratio is around 14). Compared with more classical wing sections, this kind of section does not undergo as dramatic a stall for high angles, and the lift coefficient remains high even for angles up to $30^{\circ}$.

At low $R e$ and $\alpha<3^{\circ}$, the stagnation point is on the upper wall and the separated flow below the section is accelerated more than above, corresponding to the observed downward lift. The equivalent Rankine body representing the solid body plus the separated region would show a sort of elliptic shape slightly tilted downward with respect to the incoming flow, which corresponds to the moderate and negative lift and high drag observed. For higher $R e$ in the same range of $\alpha$, transition of the boundary layer on the upper wall allows for the flow to stay attached much farther downstream on the upper wall. The bound circulation and related upwash in front of the body move the stagnation point close to the leading edge, faster flow is above the section, and the recirculation under the profile is more restricted to the cavity into the arc. The associated jump to an upward lift is described in detail in Ref. [20]. It may be considered that this high-camber section at moderate angles behaves as a rather bluff body at low $R e$ and as a more slender lifting body at higher $R e$.

For the two highest $R e$ values, the polar curve exhibits a sort of double stall. The first stall takes place in a range from $6^{\circ}$ up to $11^{\circ}$ and corresponds to an increasing rear separation on the convex upper wall due to excessive adverse pressure gradient. However, the separation point does not progress all the way to the leading edge. The second stall occurs around $24^{\circ}$, where the flow on the suction side separates right at the sharp leading edge. This behavior might be comparable to the one observed for thick airfoils used in wind turbine applications where a quasi-linear growth of $C_{L}$ is followed by a first decrease, then a plateau, and finally deep stall when separation occurs at the leading edge (see, e.g., Ref. [25]).

The jump in $C_{L}$ observed at $\alpha=15^{\circ}$ for $R e=6.82 \times 10^{4}$ may be related to the formation of a small leading edge separation bubble as suggested by the curvature of streamlines above the leading edge visible for $\alpha \geq 18^{\circ}$. A similar flow pattern suggesting the occurrence of a flow separation at the leading edge is also visible for the two higher $R e$ values (Fig. 8) showing no jump in $C_{L}$. However, the present PIV measurements do not allow for accurately determining the occurrence of separation at the leading edge, and this would need further investigation and refined measurements around the leading edge. Moreover, this jump in $C_{L}$ resembles the one observed by Chen et al. [26] on a slightly cambered plate with rounded nose at low $R e$. Their simulations, consistent with experimental results from Pelletier and Muller [27] show a sudden jump in $C_{L}$ (increased slope) at $5^{\circ}$ angle of attack associated to the growth of a laminar separation bubble.

For the particular section studied in this paper, it is not easy to strictly define an ideal angle of attack in the sense of zero separation at the leading edge because of the corner behind the leading edge on the lower surface due to the plate thickness. Nevertheless, an approximate ideal angle of attack may be estimated to be around $16^{\circ}$ on this section but may well depend on the $R e$.

For $R e=6.82 \times 10^{4}$, the angle of attack where $C_{L}$ changes from negative to positive corresponds to the appearance of a stagnation point on the lower wall near the trailing edge, and the disappearance of the small secondary vortex in front of the recirculation inside the cavity. However, no particular change in $d C_{L} / d \alpha$ is visible.

For angles higher than around $7^{\circ}$ or $8^{\circ}$, increasing rear separation on the suction side corresponds to lowering the $C_{L}$ growth (low $R e$ ) or decreasing $C_{L}$ (high $R e$ ). Then for higher angles, it is possible that a leading edge separation bubble corresponds to the observed $C_{L}$ jump. Once again, this would need a refined investigation around the leading edge.

\section{Conclusions}

Variations of the lift and drag forces and associated flow patterns around a circular arc section with $22.3 \%$ camber and sharp leading edge in a nominally $2 \mathrm{D}$ flow have been investigated in a wide range of angles of attack for three values of the $R e$ across the transitional range. Despite the simple geometry, this flow is rather complex because of high pressure gradients, high curvature, and massive separation. The presented results help to better understand this kind of flow and how the fluid forces relate to the flow pattern. Moreover, this dataset gives an experimental benchmark to compare with, particularly interesting to assess transition models as dramatic $R e$ effects are evidenced. This more or less slender body can generate high lift (at the expense of a high drag). For a low $R e$, a jump in $C_{L}$ is evidenced at $\alpha=15^{\circ}$, whereas at higher $R e$, a first stall is followed by an upsurge in $C_{L}$ at $\alpha$ around $10^{\circ}$. Such a high-camber plate gives a simple high-lift device easy to manufacture, which might be interesting for low-cost wind or tidal stream energy-harvesting systems. Finally, the sudden jump in lift with $R e$ at constant and low angles of attack may be used for practical applications such as a velocity threshold detector, or the passive stability control of an unmanned flying vehicle, as proposed in Ref. [28].

\section{Acknowledgments}

The author is grateful to the French Naval Academy Research Institute technical staff, to Alessandro Lombardi and Victor Martin for their help in the experimental work and data processing. Fruitful 
discussions with Marc Rabaud, Peter Richards, Richard Flay, and Ignazio Viola are also acknowledged.

\section{References}

[1] Bot, P., Viola, I. M., Flay, R. G. J., and Brett, J.-S., "Wind-Tunnel Pressure Measurements on Model-Scale Rigid Downwind Sails," Ocean Engineering, Vol. 90, Nov. 2014, pp. 84-92. https://doi.org/10.1016/j.oceaneng.2014.07.024

[2] Richards, P. J., Johnson, A., and Stanton, A., "America's Cup Downwind Sails-Vertical Wings or Horizontal Parachutes?" Journal of Wind Engineering and Industrial Aerodynamics, Vol. 89, Nos. 14-15, 2001, pp. $1565-1577$. https://doi.org/10.1016/S0167-6105(01)00135-0

[3] Collie, S., "Application of Computational Fluid Dynamics to TwoDimensional Downwind Sail Flows," Ph.D. Dissertation, Yacht Research Unit, Univ. of Auckland, Auckland, 2006

[4] Sunada, S., Yasuda, T., Yasuda, K., and Kawachi, K., "Comparison of Wing Characteristics at an Ultralow Reynolds Number," Journal of Aircraft, Vol. 39, No. 2, 2002, pp. 331-338. https://doi.org/10.2514/2.2931

[5] Laitone, E. V., "Aerodynamic Lift at Reynolds Numbers Below $7 \times 10^{4}, "$ AIAA Journal, Vol. 34, No. 9, 1996, pp. 1941-1942. https://doi.org/10.2514/3.13329

[6] Ramasamy, M., Johnson, B., and Leishman, J. G., "Understanding the Aerodynamic Efficiency of a Hovering Micro-Rotor," Journal of the American Helicopter Society, Vol. 53, No. 4, 2008, pp. 412-428. https://doi.org/10.4050/JAHS.53.412

[7] Hein, B. R., and Chopra, I., "Hover Performance of a Micro Air Vehicle: Rotors at Low Reynolds Number," Journal of the American Helicopter Society, Vol. 52, No. 3, 2007, pp. 254-262. https://doi.org/10.4050/JAHS.52.254

[8] Nava, S., Bot, P., Cater, J., and Norris, S., "Modelling the Lift Crisis of a Cambered Plate at $0^{\circ}$ Angle of Attack," 20th Australasian Fluid Mechanics Conference, Australasian Fluid Mechanics Soc., Perth, Australia, Dec. 2016.

[9] Zhang, L., and Agarwal, R. K., "Numerical Simulation of Flow Past a Circular Arc and a Truncated Circular Cylinder in Transitional Flow," AIAA SciTech Forum, 2018 AIAA Aerospace Sciences Meeting, AIAA Paper 2018-0820, Jan. 2018.

[10] Nagapetyan, H., and Agarwal, R. K., "Application of the WA- $\gamma$ Transition Model for Flow Past a Circular Arc," AIAA Scitech Forum 2019, AIAA Paper 2019-2323, Jan. 2019. https://doi.org/10.2514/6.2019-2323

[11] Bruining, A., "Aerodynamic Characteristics of a Curved Plate Airfoil Section at Reynolds Numbers 60,000 and 100,000 and Angles of Attack from -10 to 90 Degrees," Dept. of Aerospace Engineering, Delft Univ. of Technology Rept. LR-281, Delft, The Netherlands, 1979.

[12] Okamoto, M., and Azuma, A., "Experimental Study on Aerodynamic Characteristics of Unsteady Wings at Low Reynolds Number," AIAA Journal, Vol. 43, No. 12, 2005, pp. 2526-2536. https://doi.org/10.2514/1.14813

[13] Tezuka, A., Sunada, Y., and Rinoie, K., "Surface Pressure Distributions on 4\% Circular Arc Airfoil at Low Reynolds Number," Journal of Aircraft, Vol. 45, No. 6, 2008, pp. 2164-2167. https://doi.org/10.2514/1.35365

[14] Milgram, J. H., "Section Data for Thin, Highly Cambered Airfoils in Incompressible Flow," NASA CR-1767, 1971.

[15] Sato, M., Asada, K., Nonomura, T., Kawai, S., and Fujii, K., "LargeEddy Simulation of NACA 0015 Airfoil Flow at Reynolds Number of
$1.6 \times 10^{6}$," AIAA Journal, Vol. 55, No. 2, 2017, pp. 673-679. https://doi.org/10.2514/1.J054963

[16] Hristov, G., and Ansell, P. J., "Poststall Hysteresis and Flowfield Unsteadiness on a NACA 0012 Airfoil," AIAA Journal, Vol. 56, No. 7, 2018, pp. 2528-2539. https://doi.org/10.2514/1.J056774

[17] Collie, S., Jackson, P., and Gerritsen, M., "Validation of CFD Methods for Downwind Sail Design," High Performance Yacht Design Conference, Royal Inst. of Naval Architects, London, 2002.

[18] Miklasz, K., LaBarbera, M., Chen, X., and Socha, J. J., "Effects of Body Cross-Sectional Shape on Flying Snake Aerodynamics," Experimental Mechanics, Vol. 50, No. 9, 2010, pp. 1335-1348. https://doi.org/10.1007/s11340-010-9351-5

[19] Holden, D., Socha, J. J., Cardwell, N. D., and Vlachos, P. P., "Aerodynamics of the Flying Snake Chrysopelea Paradisi: How a Bluff Body Cross-Sectional Shape Contributes to Gliding Performance," The Journal of Experimental Biology, Vol. 217, No. 3, 2014, pp. 382-394. https://doi.org/10.1242/jeb.090902

[20] Bot, P., Rabaud, M., Thomas, G., Lombardi, A., and Lebret, C., "Sharp Transition in the Lift Force of a Fluid Flowing Past Nonsymmetrical Obstacles: Evidence for a Lift Crisis in the Drag Crisis Regime," Physical Review Letters, Vol. 117, No. 23, Dec. 2016, Paper 234501. https://doi.org/10.1103/PhysRevLett.117.234501

[21] Stevens, P. R. R. J., Babinsky, H., Manar, F., Mancini, P., Jones, A. R., Nakata, T., Phillips, N., Bomphrey, R. J., Gozukara, A. C., Granlund, K. O., et al., "Experiments and Computations on the Lift of Accelerating Flat Plates at Incidence," AIAA Journal, Vol. 55, No. 10, 2017, pp. 32553265.

https://doi.org/10.2514/1.J055323

[22] Zheng, J., Cui, Y. D., Zhao, Z., Li, J.-M., and Khoo, B. C., "Flow Separation Control over a NACA 0015 Airfoil Using NanosecondPulsed Plasma Actuator," AIAA Journal, Vol. 56, No. 6, 2018, pp. 2220-2234. https://doi.org/10.2514/1.J056111

[23] Marchand, J. B., "Experimental Analysis and Simulation of Foil Sections for Tidal Turbine Application,” Ph.D. Dissertation, Institut de Recherche de l'Ecole Navale, ENSAM, Paris, 2014.

[24] "DynamicStudio User's Manual," Dantec Dynamics S/A, Denmark, 2013, pp. 220-226, Sec. 15.3, Chap. 15.

[25] Timmer, W. A., and van Rooij, R. P. J. O. M., "Summary of the Delft University Wind Turbine Dedicated Airfoils," Journal of Solar Energy Engineering, Transactions of the ASME, Vol. 125, No. 4, 2003, pp. 488-496. https://doi.org/10.1115/1.1626129

[26] Chen, Z. J., Quin, N., and Nowakowski, A. F., "Three-Dimensional Laminar-Separation Bubble on a Cambered Thin Wing at Low Reynolds Numbers," Journal of Aircraft, Vol. 50, No. 1, Jan.-Feb. 2013, pp. $152-163$. https://doi.org/10.2514/1.C031829

[27] Pelletier, A., and Muller, T. J., "Low-Reynolds Number Aerodynamics of Low-Aspect-Ratio, Thin/Flat/Cambered-Plate Wings," Journal of Aircraft, Vol. 37, No. 5, 2000, pp. 825-832. https://doi.org/10.2514/2.2676

[28] Bot, P., Ouest Valorisation SAS, French Patent Application for a "Transducteur adapte a la generation de forces en function de la vitesse d'ecoulement d'un fluide," No. fr15/01418, 2015, filed 3 July 2015 , Extended to International Patent Application PCT/FR2016/051685, 2016. 